\section{APUNTES PARA UNA SOCIOLOGÍA DEL CÓMIC}

\author{
Jordi Giner Monfort \\ Universitat de València \\ https://orcid.org/0000-0001-6899-3609
}

Jordi.Giner@uv.es

\section{SKETCHES FOR A SOCIOLOGY OF COMICS}

Cómo citar este artículo/Citation: Giner Monfort, Jordi (2021). Apuntes para una sociología del cómic. Arbor, 197(801): a614. https://doi.org/10.3989/arbor.2021.801004

Recibido: 24 marzo 2021. Aceptado: 6 mayo 2021. Publicado: 9 diciembre 2021.

RESUMEN: Este artículo se centra en el análisis de los estudios teóricos y empíricos sobre la sociología del cómic durante los siglos XX y XXI. Aunque en la actualidad el cómic está ampliamente aceptado en el ámbito académico, tanto en publicaciones periódicas como en monografías especializadas, esto no ha sido lo habitual en el pasado. Los primeros estudios fueron excepciones en el ámbito más amplio de la sociología de la comunicación, y no será hasta los años 50 cuando se popularicen en la academia. Uno de los catalizadores de la sociología del cómic fue la obra de Umberto Eco (1968), a partir de la cual llegarían estudios más profundos como los de Dorfman y Mattelart (1971) o Luc Boltanski (1975). Finalmente, se analizan trabajos empíricos que toman como base el lenguaje del cómic.

PALABRAS CLAVE: Sociología de los medios de comunicación; sociología del cómic; audiencias; público lector.
Copyright: (C) 2021 CSIC. Este es un artículo de acceso abierto distribuido bajo los términos de la licencia de uso y distribución Creative Commons Reconocimiento 4.0 Internacional (CC BY 4.0).

ABSTRACT: This article focuses on the analysis of theoretical and empirical studies on the sociology of comics during the twentieth and twenty-first centuries. Although currently comics are widely accepted in academia, both in journals and specialised monographs, this was not common in the past. The first studies were exceptions in the broader scope of communication sociology, and they only became popular in academia as of the 1950s. One of the catalysts for the sociology of comics was Umberto Eco (1968), from which deeper studies would come, such as those by Dorfman and Mattelart (1971) or Luc Boltanski (1975). Finally, some empirical research based on the language of comics is analysed.

KEYWORDS: Sociology of mass media; sociology of comics; audiences; reading public. 


\section{INTRODUCCIÓN}

La historieta o cómic, como medio de comunicación, se desarrolla como la conocemos hoy en día durante el siglo $X X$, al igual que otros medios de comunicación. Pese a que los antecedentes más o menos directos del cómic se remonten a miles de años, lo que incluiría desde las pinturas prehistóricas hasta las biblias pauperum pasando por tapices seriados como el de Bayeux (Hogben, 1949), no será hasta finales del siglo XIX cuando alcance la forma con la que se identifica actualmente, básicamente a partir de la obra de ilustradores como Töpffer, Outcault, Mestres, Cilla y un largo etcétera (Sabin, 1996; Martín, 2011).

A diferencia de otros medios de comunicación, el cómic no supone un avance en la técnica que suponga un salto cualitativo, como en el caso de la radio y la televisión, sino una evolución lógica de aquello que ya se venía utilizando desde hacía siglos, es decir, la reproducción impresa de imágenes seriadas. Es por ello por lo que la sociología se fijó en el cómic cuando se manifestó una confluencia de circunstancias determinadas: que el cómic alcanzara una popularidad significativa con tiradas de millones de ejemplares; que esta se diera en el contexto geográfico de los Estados Unidos; y que ello coincidiera con la expansión de la sociología en el ámbito académico, y en particular de las orientaciones cuantitativas propias del inicio de siglo, especialmente de las que se centraban en el análisis de los medios de comunicación como la obra de Paul Lazarsfeld (1940) o Robert K. Merton (1946) ${ }^{1}$. No obstante, el cómic ha sido considerado históricamente como un campo menor, mayoritariamente vinculado con el público infantil o juvenil, prácticamente hasta el final del siglo XX, lo que le ha acarreado ciertos prejuicios en el ámbito académico (Berger, 1970; Groensteen, 2008; Lent, 2010). A pesar de ello, prácticamente desde mediados de los años 90 del siglo XX emerge un campo de estudios sobre los cómics que es heredero, entre otros, de Eco, Boltanski o Dorfman.

Dentro de esta corriente de estudios sobre el cómic, que eclosionará en la segunda década del siglo XXI, se puede localizar un interés de la sociología por el medio, orientada tanto al aspecto comunicativo, esto es las audiencias, como a los efectos sobre el público lector, pasando por las condiciones de producción y reproducción o la manera en que el medio trasciende su propio campo en el sentido más estricto. Este escrito intenta acometer un análisis de la producción académica relacionada con la sociología del cómic con un doble objetivo: el primero, de divulgación y ordenación del corpus teórico y empírico a nivel nacional e internacional. El segundo, la legitimación del campo del cómic en el ámbito de la sociología académica. Para ello se ha realizado una revisión bibliográfica extensa e interdisciplinar, puesto que algunos de los enfoques pueden contener elementos de interés para la sociología a pesar de estar razonados desde disciplinas como la psiquiatría o la antropología.

Organizamos el análisis en torno a dos ejes principales: el primer eje es el del análisis del medio de comunicación desde la óptica de la sociología, que empieza ya en los años 40 del siglo XX en los Estados Unidos de América y finaliza con la eclosión de revistas académicas interdisciplinares centradas en el análisis del medio y la publicación de monografías en las editoriales de mayor prestigio. Las principales escuelas de análisis de los medios de comunicación se han fijado en algún momento en el fenómeno del cómic, desde el funcionalismo al modelo de conflicto social pasando por los paradigmas interaccionistas y los estudios culturales. El segundo eje es el del reconocimiento del cómic como un método de recogida de información de carácter empírico al mismo nivel que otros productos culturales como noticias, artículos, documentales, etcétera.

\section{APROXIMACIÓN BIBLIOGRÁFICA A LA SOCIO- LOGÍA DEL CÓMIC}

De acuerdo con Thompson (1990), el análisis de cualquier objeto cultural debería centrarse en tres aspectos principales, lo que él denomina la aproximación tripartita: primero, la producción y trasmisión de formas simbólicas, lo que sería el ámbito de la semiótica; segundo, la construcción de los mensajes y, por tanto, su contenido; y tercero, la recepción y apropiación de los mensajes, esto es, el estudio de las audiencias. En este sentido, los estudios literarios sobre el cómic se suelen centrar en el segundo aspecto, mientras que las aproximaciones desde lo social se centran

1 Aunque nunca se llegaron a publicar, Merton dirigió dos estudios sobre los efectos de las tiras cómicas en el combate contra los prejuicios raciales en el contexto del Bureau of Applied Social Research (BASR) de la Columbia University. Berelson también dirigió un estudio sobre la aceptación de los sindicatos a partir de las tiras cómicas de The Story of Labor y Patricia Kendall hizo lo propio para el American Jewish Committee a partir de las tiras de Mr. Bigott (Simonson, 2006). De los cuatro estudios solamente conocemos uno de ellos (Kendall y Wolf, 1949). Lo que parece evidente es que había un gran interés en la investigación sobre el cómic en el BASR. 
en el primer y tercer apartado, esto es, las formas simbólicas y las audiencias, a pesar de que posiblemente la mejor aproximación sea un análisis holístico del medio (Brienza, 2010). Organizamos la exposición del corpus teórico partiendo de las audiencias, para tratar después los contenidos y las formas simbólicas y terminar con la legitimación académica del cómic.

\subsection{Las audiencias}

Los primeros trabajos sobre el cómic se centran en el apartado de la recepción, en un momento histórico en el que los estudios parciales de los medios de comunicación se fijaban en las audiencias y en los efectos de los mensajes sobre estas. Este tipo de investigación aparece en los Estados Unidos durante los años 40 del siglo XX, prácticamente al mismo tiempo que se popularizaba el medio con The Funnies (1931) o Superman (1938), y de manera paralela a la investigación centrada en otros medios de comunicación (radio, revistas, cine, etcétera). Así, los primeros estudios sobre el cómic parten de una óptica puramente descriptiva de la realidad.

Puede que uno de los primeros estudios sobre el cómic, dejando aparte las primeras referencias teóricas (Tilley, 2017) sea el de George Hill y Estelle Trent (1940) que analiza el interés y las prácticas lectoras de 256 niños y niñas de Filadelfia de 10 a 12 años a partir de un cuestionario que aplicaron en 1938. Para ello, se centran en las tiras cómicas que generaban mayor atención y observan diferencias significativas entre las preferencias lectoras por grupo étnico y sexo y también una mayor inclinación al cómic de acción en los niños. Paul Witty (1941a) iría más allá y llegó a reunir una muestra de 334 niños y niñas de 10 a 12 años a los que interrogó sobre el número de que cómics leían y sus títulos ${ }^{2}$. En otro estudio publicado en la misma fecha profundizaría sobre una muestra aleatoria, no solo en la frecuencia y los gustos en materia de cómic, sino también en la inclinación a la producción de cómics por parte del alumnado encuestado (Witty, 1941b). En la misma dirección, Ruth Strang se centró en los factores que favorecen la lectura, para lo que entrevistó a 30 niños y niñas y analizó 150 informes escolares (Strang, 1943). Uno de los factores que identificó fue la circulación de cómics de mano en mano, lo que aumentaba su potencial audiencia, e incluso dio a conocer un mercado de intercambio en modo cooperativo. Strang, contrariamente al ambiente prohibicionista que llegaría una década más tarde, proponía integrar el cómic en la escuela, dado su papel de atracción hacia la lectura, lo que parecía cuadrar con otros estudios similares que, sin duda, debía conocer (Thorndike, 1941). Aunque con una muestra menor, el estudio de Katherine Wolf y Marjorie Fiske (1949), basado en 104 entrevistas a niños y niñas, ayudó a definir tres tipos de lectores de cómic: fans (37\% de la muestra), lectores ocasionales (48\%) e indiferentes y hostiles (15\%). Wolf y Fiske observan de qué manera diferente tipo de material es utilizado por distintas franjas etarias: los cómics de animales son útiles en cuanto que resolverían sus necesidades proyectivas tempranas, mientras que los de superhéroes satisfarían sus egos. Asimismo, destacan el carácter realista de los ambientes urbanos presentes en series como Superman o Batman, en tanto que los héroes correspondientes de la literatura clásica viven en entornos irreales o que serían menos fácilmente identificables para niños y niñas. Asímismo, Patricia Kendall y Katherine Wolf tratan de dilucidar los efectos de la lectura de cómics sobre los prejuicios raciales (Kendall y Wolf, 1949). Para ello, aplican entrevistas en profundidad (de una a tres horas de duración) a 160 hombres blancos de clase obrera, focalizando la atención en su reacción a los cómics de $\mathrm{Mr}$ Bigott ${ }^{3}$, un personaje de clase media con claros prejuicios raciales. Su investigación desveló que una gran parte de la muestra malinterpretaba los mensajes satíricos y creía que se trataba de un cómic que pretendía extender el racismo, en lo que profundizan a partir del proceso de interpretación.

La llegada del ambiente de represión contra los cómics de los años 50 y la subsiguiente implantación del Comics Code, como veremos a continuación, provocó el descenso en las ventas, pero también las investigaciones sobre el público lector. Prácticamente hasta los años 90 no vuelven a publicarse análisis de audiencias sobre el cómic. Estos son los casos de los estudios sobre comunidades de fans (fandom) dirigidos por Frazer (1987), Brown (1997), Pustz (1999) y Woo. El estudio de Elizabeth Frazer se centra en la lectura de los álbumes de Jackie por parte de chicas adolescentes del Reino Unido (Frazer, 1987). Se basa en siete grupos de discusión alrededor de una historieta en formato fotográfico y de la revista en cuestión para

2 No deja de ser significativo el hecho de que el trabajo de campo se realizaría poco después de la aparición de Superman, y que ya fuera este uno de los preferidos por los niños y niñas que responden a la encuesta.

3 Bigott designa, literalmente, a alguien fanático u obstinado en sus creencias. 
observar qué efectos tienen en las lectoras. Su conclusión es que el discurso, tal y como está planteado en la fotohistorieta, no es interpretado de manera unívoca, ni siquiera por el grupo que más cerca está de la clase social objetiva de sus protagonistas. Para Frazer, tratar de ideología a partir de los textos es, en la práctica, poco recomendable, dado que la interpretación varía mucho en función de la persona receptora. Brown apunta al capital cultural que desarrollan los lectores-coleccionistas y el reconocimiento dentro del grupo de iguales (Brown, 1997). Por otra parte, Pustz se basa en un trabajo de observación participante en librerías especializadas y convenciones para tratar de caracterizar el fenómeno fandom desde el punto de vista social y de consumo (Pustz, 1999). Precisamente Benjamin Woo siguió la estela de Pustz y Brown, con trabajo de campo en tiendas especializadas y convenciones, para clasificar la comunidad fandom entre completistas, aficionados y especuladores y, por otra parte, lectores, cada uno de ellos con una interpretación diferente de qué son y qué significan los cómics y con unas prácticas culturales y de consumo que son las que validan su pertenencia a la comunidad fan (Woo, 2011; Woo, 2012).

\subsection{El contenido}

Si hay una perspectiva de análisis que se ha impuesto, esta es el análisis de contenido. A pesar de los primeros escritos a favor del cómic, las investigaciones críticas, como veremos a continuación, serán más populares. Estos estudios se suelen situar en la línea de la teoría de la aguja hipodérmica, por la que se da por cierta la asunción unívoca del mensaje recibido (Laswell, 1927). Uno de los escasos ejemplos de análisis de contenido en positivo es el estudio de Thorndike sobre el número de palabras que contenían varios álbumes (dos de Superman y uno de Batman y Detective Comics) y en cuánto superaban a las mil primeras que aparecían en un manual para profesores que contaba con 20.000 . El resultado es muy positivo, ya que de las 10.000 palabras que identifica en términos medios en cada cómic, al menos 1.000 no aparecen en el listado de referencia (Thorndike, 1941). En esta línea está el estudio de Bender y Lourie sobre los efectos de la lectura de cómics en niños y niñas en el que se observó que los posibles conflictos con el cómic eran producto de la mente adulta, con lo que anticipaban el movimiento anticómic de los años 50. De hecho, Bender y Lourie recomendaban la lectura de cómics incluso a aquellos niños que tuvieran problemas de comportamiento (Bender y Lourie, 1941). Unos años más tarde Florence Heisler analizó los hábitos de lectura de 600 niños y niñas de Nueva York (1946), tanto lectores de cómics como no lectores, y comparó sus notas mediante pruebas estandarizadas (test de Standford de lectura; test de California sobre madurez mental y de personalidad) y otras medidas como el número de libros disponibles en el hogar. Heisler llegó a la conclusión de que no había diferencias significativas entre las notas de los dos grupos analizados.

Por el contrario, una de las primeras aproximaciones académicas en negativo fue la que Henry E. Schultz (1949) dedicó a la censura en el cómic, que anticipó la primera ola de estudios conservadores sobre el medio, crítica tanto con el mensaje como con el componente icónico. No obstante, el primer artículo académico con base empírica fue el que Thomas Hoult dedicó a la lectura de cómics en delincuentes juveniles. Hoult comparó la lectura de cómics en 235 jóvenes de Los Ángeles, entre los que había delincuentes y no delincuentes, y observó que los primeros habían leído más cómics de los que él mismo clasificó como lesivos y, en términos generales, más cómics que las personas sin antecedentes penales (Hoult, 1949). Pero si hay una obra académica de influencia en la historia del cómic quizá sea la del psiquiatra Fredric Wertham (1954). Wertham alerta de la violencia y las escenas sexuales más o menos explícitas presentes en los cómics con lo que analiza lo manifiesto -lo icónico- pero también lo subliminal, en ocasiones hasta límites que rozan lo increíble. Su análisis desembocaba, nuevamente, en los efectos negativos de la lectura de cómics, entre otros: analfabetismo, fantasías insanas, ideas criminales o delincuencia (Wertham, 1954: 118), lo que desencadenaría una histeria anti-comic, primero, y más tarde la implementación de un código de autoregulación de la industria editorial estadounidense, el Comics Code (Hajdu, 2009).

Otros estudios posteriores se han centrado en aspectos ideológicos, especialmente desde el trabajo de Barker (1989). Este es el caso de Vidal, que se centra en el análisis ideológico en Tintín, Flechas y Pelayos o Flash Gordon (Vidal, 1999) y más tarde en la función social de los superhéroes, como la idea de seguridad y protección en los años 30 a partir de la figura de Superman (Vidal, 2002). En los últimos años son varios los ejemplos de análisis sobre el universo Disney des-

4 Cuyas teorías, expuestas en varias revistas, ya fueron puestas en duda incluso antes de que publicara su monografía (Thrasher, 1949). 
de una vertiente crítica (Smoodin, 1994; The Project on Disney, 1995), aunque probablemente el de mayor repercusión sea el del pedagogo Henry Giroux (2001). El universo Disney, implantado globalmente, atenta contra la capacidad de oposición, algo que practicaría en todas las facetas corporativas, desde lo comunicativo a lo educativo, pasando por el marketing y también en el mensaje incrustado en, por ejemplo, sus películas más taquilleras (Giroux, 2001).

El análisis de contenido ha sido una faceta en la que se han especializado los análisis de tipo artístico y literario. Algunos artículos recientes sí apuestan por un análisis de contenido del cómic desde la perspectiva de la sociología visual (Harper, 2012). Este es el caso de la investigación de Nathan Stephens Griffin sobre el veganismo, en la que toma como método el cómic autobiográfico, a partir del cual analiza tanto el apartado discursivo como el aspecto visual (Stephens Griffin, 2019).

\subsection{Las formas simbólicas}

Pero no todo el análisis de contenido del cómic fue catastrofista. Este es el ejemplo -breve, pero de cierta entidad- de la obra de Marshall McLuhan. Su perspectiva podría caracterizarse como la primera que se fija no tanto en los efectos sobre las audiencias o el contenido, sino más bien en el análisis de la producción y transmisión de formas simbólicas mediatizadas, en este caso, por el cómic. Se centra en clásicos de las tiras cómicas publicadas en la prensa diaria como $\mathrm{Li}^{\prime} \mathrm{l}$ Abner, Little Orphan Annie, Bringing Up Father o Blondie (McLuhan, 1951). De la primera serie destaca su carácter realista, lejos del humor que suele caracterizar al cómic y el hecho de que con un único personaje simboliza la imagen del estadounidense medio. De la segunda, resalta el paralelismo con las tesis de Margaret Mead sobre la necesidad de superar a los progenitores para demostrar la propia valía. En Bringing Up Father se centra en el efecto imitación de las clases medias por parte de una familia de inmigrantes. Y por último, sobre Blondie, enfatiza la diferencia entre las primeras generaciones de inmigrantes europeos llegados a los Estados Unidos y las nuevas generaciones nacidas y criadas allí en cuanto a la probabilidad de lograr el éxito en sus carreras. También en este sentido, Riesman, Glazer y Denney (1950) utilizan el cómic en una monografía sobre la transformación de la sociedad norteamericana y cómo los agentes socializadores más allá de la familia, la escuela y el grupo de iguales -en este caso, el cómic- orientan el comportamiento individual. Reuel Denney analizó posteriormente las transformaciones sociales en la sociedad norteamericana a partir de la serie Gasoline Alley. En ella, analiza cómo el vehículo pasó de ser un mero medio de transporte a una forma diferenciada de representación social por la vía de la transformación personalizada (Denney, 1957).

En los años 70 llegan las primeras muestras de análisis crítico sobre el medio. Este es el caso de la obra de Dorfman y Mattelart (1971), quizá una de las más conocidas en su campo, sobre el Pato Donald. En ella analizan su naturaleza imperialista, alejada de lo que se supone una historia inocente para el público infantil, de tal manera que serviría de correa de transmisión de los valores capitalistas norteamericanos propios de la corporación Disney: consumismo, estereotipos sobre países en desarrollo y mensajes anticomunistas. Entre otros indicios, señalan la práctica ausencia de formas de producción, la sustitución de los conflictos de carácter social por enfrentamientos entre el bien y el mal y la simplificación de los países exóticos. Más aún, los personajes de Disney representarían una única clase, de carácter utópico, que parece querer volver a las civilizaciones anteriores a la urbanización para justificar su posición en términos de progreso y adquisición de poder. Dos años más tarde, Dorfman y Jofré indagaron, en la misma línea, sobre El Llanero Solitario y Superman en una monografía que, como la anterior, fue secuestrada por la dictadura chilena (Dorfman y Jofré, 1974).

Es de interés el análisis crítico que realiza Oscar Steimberg, desde el ámbito latinoamericano, sobre la reproducción de la ideología en Patoruzú, uno de los personajes más emblemáticos de la historieta argentina. Steimberg no solo analiza el personaje, sino también el soporte en el que se edita (la tapa, el uso del color en la historieta) e incluso los editoriales que se dedicaban en los primeros números de 1936 . Frente a un Patoruzú inocente, Steimberg sitúa los lugares comunes del perjuicio racial y nacional y sus enemigos étnicos: el judío, el gitano o el pacto turco-chino-indio (Steimberg, 1982).

A medio camino entre el análisis crítico y los Cultural Studies, Umberto Eco utilizó los cómics para su análisis estructuralista de la cultura de masas (Eco, 1968), especialmente la obra de Steve Canyon y Superman. Respecto al último, Eco apunta el hecho de que se trate al mismo tiempo de un ciudadano medio insignificante (Clark Kent) y un superhéroe con todo tipo de poderes. Su figura mítica define el contenido 
de las historias que vive y el universo temporalmente estático en el que está inmerso. Este se rige por un esquema iterativo y una ubicación espaciotemporal que no es real lo que, de alguna manera, garantiza la existencia del superhéroe e impedirá su muerte. Respecto de Steve Canyon, Eco se fija en la función social que podía cumplir para con sus millones de lectores en su formato de tira diaria. El mensaje que transmitía, los personajes que presentaba e incluso las aventuras que vivía podían ser funcionales para el imaginario americano, como el hecho que se aliste en el ejército en plena Segunda Guerra Mundial.

La escuela semiológica de Eco dejó huella en un grupo de estudiosos españoles de finales de los años 60 y principios de los 70. Puede que el primero de ellos sea Luis Gasca, que en 1966 publicó una monografía sobre el tebeo que vería su continuación solo un año después en un artículo sobre la influencia del cómic en la publicidad (Gasca, 1966; Gasca, 1967). Además de explorar la interacción entre cómic, cine, publicidad y arte, Gasca también se centra en la utilización didáctica del cómic. Una atención especial merece el prematuro ensayo de Terenci Moix (1968) dedicado al cómic. Aunque no se trate de un texto canónicamente sociológico, las referencias a autores, teorías y conceptos son constantes, como también lo es su relación con la obra de Eco. Uno de los aspectos centrales en su obra es, precisamente, el proceso de legitimación y aceptación del cómic por el mundo académico e intelectual, algo que adelanta unos años el estudio sobre el medio de Boltanski (1975). Moix también se cuestiona la recepción del cómic por el público infantil de diferentes clases sociales, con especial atención a la situación en Catalunya antes y después de la Guerra Civil, tanto en relación con el contenido, como con el formato. En este sentido, por ejemplo, propone una interpretación de las historietas de Doña Urraca en clave de transmisión de las calamidades sociales de la postguerra. Más aún, critica el inmovilismo del cómic burgués anterior a la guerra (caso de Patufet), el excesivo papel de defensa de la ideología del régimen franquista de cómics como El guerrero del antifaz y también cuestiones como el precio de los tebeos, la transmisión de los roles de género o la interacción con otros elementos del campo de los medios de comunicación (televisión, radio, cine, etc.).

El ensayo de Arthur Asa Berger (1970) sobre Li'l Abner es el primer estudio monográfico de una serie. Berger defiende la amplitud del campo del cómic y su dificultad de análisis, por lo que escoge un clásico del cómic americano, sobre el que despliega un corpus metodológico y analítico que pasa por la imagen, el texto, los segundos significados o las funciones manifiestas y latentes. Li'l Abner constituiría una sátira manifiesta de la sociedad norteamericana, al mismo tiempo que estimularía a los lectores masculinos para hacerles sentirse, de manera latente, superiores al hillbilly que representa Abner, de clase baja y escaso nivel educativo, un antihéroe, lo opuesto al sueño americano.

La irrupción del cómic en el ámbito académico a partir de los años 90 se debe, en parte, a los Cultural Studies, que legitiman el estudio de las expresiones subculturales juveniles, entre las que aparece de manera indirecta el cómic. Un ejemplo es el trabajo de Martin Barker (1989) sobre series populares en Reino Unido, como Action, Scream Inn, Jackie o el mismo Pato Donald. Para ello se basa en una combinación de métodos cualitativos, entre los que incluye entrevistas con autores, análisis de contenido y análisis de las cartas que el público lector enviaba a las editoriales, y también cuantitativos, en este caso en base a encuestas al público lector de Action (con una muestra de 135 personas) y Scream Inn (con una muestra de 618 personas). Barker desmenuza algunos de los análisis académicos ya existentes desde la óptica de lo psicológico y social. Es muy crítico con los estudios de determinados cómics en los que se interpreta que tienden a establecer estereotipos por razón de género o etnia, precisamente porque dichos análisis están condicionados a priori: si no aparecen determinados grupos sociales, estos estarían ausentes; si aparecen representando situaciones calamitosas por las que suelen pasar, se estaría estereotipando; y si, finalmente, aparecen en contextos normalizados, se aduce que no sería una situación realista.

Por el contrario, estos dos aspectos, el género y la etnicidad, han sido ampliamente analizados en los últimos años. En relación al primero, puede que la primera contribución en este sentido fuese la de Deborah Chavez (1985), basada en tiras cómicas que iban a tener un uso pedagógico en un programa de educación primaria impulsado por una editorial conservadora (Blondie, Family Circus y Beetle Bailey). En su estudio, Chavez comprueba que las mujeres están infrarrepresentadas, en una proporción de 5,7 hombres por cada mujer, y que cuando aparecen suelen representarse sin empleo o en oficios concretos como secretaria, frente a una exposición más diversa de oficios en el sexo masculino. También observa un ba- 
lance desigual en la asunción de roles de cuidado por sexo. En esta misma línea, otros estudios han insistido en las diferencias de género (Brabant y Mooney, 1997); género y etnia (Glascock y Preston-Schreck, 2004); coerción (Matacin y Burguer, 1987); género y manga (Ekachai y Drout, 1996); género y superhéroes Marvel (Murphy, 2016); o la representación de modelos masculinos hegemónicos (Parasecoli, 2014). Respecto al estudio de la etnicidad, una de las primeras referencias es la de Jack Shaheen acerca de las imágenes de la población árabe en los cómics americanos (Shaheen, 1994). A partir de entonces tanto la cuestión étnica como la nacional entran en la agenda de investigación. Un ejemplo del primer punto de vista es el análisis de Wonder Woman como inmigrante griega desde el punto de vista de la inserción de la inmigración (Smith, 2001). Un ejemplo del segundo punto de vista son los análisis en clave nacional o poscolonial de Asterix y Obelix o de los personajes de Jacques Tardi (Miller, 2007: 149 y ss.).

\subsection{La legitimación académica}

Siguiendo la aproximación tripartita de Thompson (1990), el estudio que más se ha acercado a un análisis holístico del cómic es el que realizó Luc Boltanski para el primer número de Actes de la Recerche en Sciencies Sociales (Boltanski, 1975). Su aproximación demuestra que el cómic francés gozaba en los años 70 de una legitimación cultural notable: había superado las primeras muestras de rechazo académico acaecidas en los años 50 y había llegado al éxito comercial con Tintin o Asterix y Obelix y revistas como Pilote. Boltanski se adentra en el cómic como objeto cultural de consumo, sin olvidar su conexión con los campos del arte y los medios de comunicación, mecanismos que dotarán tanto al medio como a sus productores de legitimidad y reconocimiento. Paralelamente, analiza una encuesta de Sofres de 1971 sobre la lectura de revistas de cómics (Pilote, Spirou, Tintin, Mickey, Pif y Echantillon), y se adentra en el análisis académico del cómic y los mecanismos de legitimación y formación del dispositivo de producción, reproducción y celebración que acompaña a la transformación del campo del cómic en hegemónico.

Se puede considerar que en los años 90 el estudio del cómic en la academia está bien asentado, aunque la cantidad de publicaciones no fuera mucho mayor que antes de la eclosión de estudios de los años 70. Según Web of Science, en los años 90 se publican unas 100 referencias académicas anuales sobre cómics, más de 200 durante la primera década del siglo XXI y se llega al máximo histórico en el año 2017, con 831 publicaciones indexadas. También será durante los años 90 cuando se encuentren los primeros readers sobre diferentes aspectos del cómic, como la ideología, las audiencias o el género. Dos factores importantes que hay que tener en cuenta para entender este viraje académico son la expansión desde los años 70 al público adulto y la llegada de la novela gráfica en los 90, con temas que anteriormente parecían vetados: la no ficción, el género autobiográfico, la política, etc.

En el siglo XXI queda patente la entrada del cómic en la academia. Las grandes editoriales académicas no solo publican obras puntuales sobre el medio, sino que le dedican colecciones. Estos son los casos de Routledge Advances in Comic Studies (2015) o Palgrave Studies in Comics and Graphic Novels (2016). Incluso se crean las primeras revistas académicas centradas en el estudio multidisciplinar del cómic, como Journal of Graphic Novels and Comics (2010); Studies in Comics (2010); Historietas. Revista de Estudios sobre la Historieta (2011) o The Comics Grid: Journal of Comics Scholarship (2013). De manera paralela, es significativa la irrupción de congresos, como The International Graphic Novel \& Comics Conference, que ya suma once ediciones; la creación de organismos de investigación como el International Manga Research Center en Kyoto; o la aparición de estudios de máster en Dundee University o en la Universitat de València. Como colofón a su legitimación académica, en 2015 se presentó una tesis doctoral en formato historieta, la primera que utilizó este medio para su defensa (Sousanis, 2015).

Por otra parte, puede que la inclusión de la perspectiva de la producción fuera la última pieza de un análisis sociológico holístico. A pesar de que la perspectiva autoral ya había sido contemplada en Boltanski (1975) e incluso si retrocedemos algo más en el caso de Moix (1968), no ha sido hasta el siglo XXI cuando se han incorporado aspectos como la autoría, los condicionantes legales, organizativos y técnicos (Brienza, 2010; Gabilliet, 2009; Brienza y Johnston, 2016).

\section{EL CÓMIC COMO MÉTODO}

Desde una óptica sociológica, se podrían añadir al menos dos vertientes más a la aproximación tripartita de Thompson (1990). La primera es el uso del cómic como elemento de apoyo y complemento del trabajo de campo empírico, especialmente en lo relacionado con el soporte visual que tradicionalmente han prota- 
gonizado fotografías, vídeos, esquemas, etcétera, que han sido ampliamente utilizados como herramientas de apoyo en el análisis sociológico y que entronca con la sociología visual (Harper, 2012). La segunda es la utilización del cómic como herramienta de análisis sociológico, tanto de temas macro como de aspectos micro, a partir de una obra ya publicada.

\subsection{El cómic como herramienta empírica}

Desde el punto de vista metodológico, el uso del dibujo o del lenguaje del cómic aporta algunas ventajas frente a otros tipos de soporte visual: el dibujo deja lugar a la imaginación de la persona lectora, incorpora la representación mental de aquello que ha sido observado y preserva el anonimato en mayor medida que la fotografía o el vídeo (Kuschnir, 2016).

El uso del lenguaje del cómic como herramienta de producción de imágenes que puedan ser utilizadas como parte del trabajo de campo y analizadas posteriormente se remonta, al menos, a 1990. La antropóloga Gillian Crowther utilizó sus dibujos para reflejar procesos creativos como los de la creación de un tótem, y al mismo tiempo entrar en la comunidad Haida de la Columbia Británica. El uso de otras técnicas visuales, como la fotografía o el vídeo, estaba vetado, por lo que se convirtió en una manera menos invasiva de reflejar lo observado (Crowther, 1990). Unos años después, Lois Ahrens y el equipo del proyecto The real cost of prisons comix analizan detalladamente el sistema penitenciario norteamericano, a partir de datos secundarios, referencias académicas y de las respuestas que ofrecen los reos a los cómics desde la prisión. Todo ello en formato cómic autoeditado (Ahrens, 2008). Otro ejemplo de la utilización del cómic para reflejar el trabajo de campo sociológico es el que Ayers y Alexander-Tanner utilizan para reflejar el sistema educativo en los Estados Unidos. En su monografía tocan varios temas de interés de la sociología de la educación: el alumnado, las pruebas de nivel, los estilos de aprendizaje o la relación entre clase social y desempeño en el aula (Ayers y Alexander-Tanner, 2013). También ejemplifican algunas entrevistas semidirigidas en formato cómic, en este caso a personas que consideran buenos docentes por sus prácticas cotidianas. La particularidad de la investigación del Oslo University College sobre el espacio público universitario, accesibilidad y diversidad es que sus resultados se presentan únicamente en formato cómic (Bartoszko, Leseth y Ponomarew, 2010). Mediante las diferentes historias que contiene el volumen se intentan reflejar algunas situaciones referentes a la comunicación y la diversidad que derivan de las diferentes técnicas de investigación implementadas, entre las que destacan la observación, la comunicación informal y las entrevistas semiestructuradas. En el caso de Michael Atkins el cómic es un recurso más de los utilizados en su tesis doctoral sobre los lugares de encuentro sexual de la comunidad gay en Manchester (Atkins, 2013). Con sus dibujos, y con la práctica habitual en etnografía de cambiar los nombres reales, consigue descripciones de lugares, personas y situaciones que nunca habría sido posible reflejar con fotografías o vídeo, además de que habría atentado contra el anonimato de sus informantes, y con ello habría cerrado posibles futuros trabajos de campo en esta comunidad. De la misma manera, Alex Pavlotski utilizó en su tesis doctoral este recurso para, entre otras cosas, reflejar las distintas posiciones ideológicas de las personas aficionadas a la práctica del parkour (Pavlotski, 2015). En este caso, el recurso al lenguaje del cómic buscaba preservar el anonimato de los informantes debido a que en muchas ocasiones la práctica del parkour roza lo ilegal, con lo que estaría comprometiendo a sus informantes. Además, recogió anécdotas de los entrenamientos y la vida cotidiana de dos aficionados australianos al parkour. Cabe destacar la utilización del cómic para recoger discursos textuales, de la misma manera que se hace en la etnografía cualitativa con los discursos registrados con grabadora o con notas del diario de campo. Otro ejemplo similar es el de Vigurs, Jones y Harris (2016) sobre la deuda generada por los estudios superiores en el Reino Unido, con dos apartados diferenciados: el apartado analítico, a partir de datos secundarios y estadísticas sobre endeudamiento estudiantil; y por otra parte el análisis de casos de estudiantes endeudados y las dificultades a las que se enfrentan. Por último, en el caso de Carroll, Witten y Calder-Dawe (2019) se analiza un proceso de investigación en una población con discapacidad dirigido a dirimir los factores que potencian o dificultan su participación en la vida comunitaria, algo que se ha publicado en formato cómic, pero también en formato tradicional en una revista académica sobre biomedicina (Carroll et al., 2018).

\section{CONCLUSIONES}

El cómic viene ocupando un lugar en la academia al menos desde el año 1941, pocos años después de su popularización en el ámbito norteamericano. A través de diferentes autores y obras se ha podido seguir un hilo académico que une diferentes perspectivas teóri- 
cas y empíricas, desde el funcionalismo hasta la utilización del cómic como material empírico autobiográfico. Han aparecido grandes nombres de la sociología que van desde McLuhan a Eco, pasando por Berger o Boltanski. Así pues, uno de los objetivos de este artículo vendría a cumplirse, en tanto que se ha podido comprobar el alto grado de legitimación del campo del cómic, especialmente en el ámbito internacional.

Si bien se puede hablar de que en la actualidad vivimos un enésimo renacimiento del cómic en el Estado español (con un incremento de las publicaciones anuales, la existencia de grupos de investigación y congresos académicos o la creación de másteres y cátedras dirigidas a su estudio y promoción), aún existe un déficit de análisis del campo del cómic desde lo sociológico. Por una parte, porque las perspectivas de análisis del contenido artístico o literario son aún mayoritarias. Por otra parte, porque la publicación de investigaciones empíricas es aún muy pobre, más aún cuando se trata de publicaciones de cierto impacto o revistas indexadas. Este fenómeno, que no es nuevo, se produce en parte por desconocimiento, en parte por una infravaloración que ya es histórica (Berger, 1970). A ello debemos sumar que el cómic en los estudios de audiencias lectoras y la elaboración de índices de lectura es aún residual: no se trata solamente de que su recuento sea bajo en estos índices, sino también su práctica ausencia como variable a considerar.

Por último, no quisiéramos terminar este análisis sin hacer mención a un fenómeno relevante que es la feminización de la investigación sobre el cómic, algo que viene siendo habitual desde el inicio del análisis académico del cómic. Se trata de investigadoras como Ruth Strang, Florence Heisler, Katherine Wolf o Marjorie Fiske que exploraron en los años 40 del siglo XX las posibilidades del cómic y su relación con la vida cotidiana de niños y niñas en los Estados Unidos y los índices de lectura.

\section{AGRADECIMIENTOS}

Al profesor y compañero José Beltrán Llavador, en agradecimiento por los ánimos y la revisión del texto preliminar y en reconocimiento de su amor por el tebeo.

\section{REFERENCIAS}

Ahrens, Lois (ed.) (2008). The real cost of prisons comix. Oakland: PM Press.

Atkins, Michael (2013). Cruising the Village: A visual ethnography of public sex between men in Manchester city centre [Tesis doctoral inédita]. Manchester: The University of Manchester.

Ayers, William y Tanner, Ryan Alexander (2013). Enseñar, un viaje en cómic. Madrid: Ediciones Morata.

Barker, Martin (1989). Comics. Ideology, Power \& the Critics. Manchester: Manchester University Press.

Bartoszko, Aleksandra; Leseth, Anne Brigitte y Ponomarew, Marcin (2010). Public Space, Information Accessibility, Technology and Diversity at Oslo University College. Oslo: Oslo University College.

Bender, Lauretta y Lourie, Reginald (1941). The effects of comic books on the ideology of children. American Journal of Orthopsychiatry, 11 (3): 540-550. https:// doi.org/10.1111/j.1939-0025.1941. tb05840.x

Berger, Arthur (1970). Li'l Abner: a study in American Satire. New York: Twaine Publishers.
Boltanski, Luc (1975). La constitution du champ de la bande dessinée. Actes de la Recerche en Sciencies Sociales, 1 (1), pp. 37- 59. https://doi.org/10.3406/ arss.1975.2448

Brabant, Sarah y Mooney, Linda (1997). Sex Role Stereotyping in the Sunday Comics: A Twenty Year Update. Sex Roles, 37 (3- 4): 269-281. https://doi. org/10.1023/A:1025604028966

Brienza, Casey (2010). Producing comics culture: a sociological approach to the study of comics. Journal of Graphic Novels and Comics, 1 (2): 105 -119. https://doi.org/10.1080/21504857.20 10.528638

Brienza, Casey y Johnston, Paddy (2016). Cultures of Comics Work. New York: Palgrave Macmillan. https://doi. org/10.1057/978-1-137-55090-3

Brown, Jeffrey (1997). Comic Book Fandom and Cultural Capital. The Journal of Popular Culture, 30 (4): 13- 31. https://doi.org/10.1111/j.00223840.1997.3004_13.x

Carroll, Penelope; Witten, Karen y Calder-Dawe, Octavia (2019). Comic explainer: young disabled New Zealanders on the barriers to a better life. The
Conversation. Disponible en https:// theconversation.com/comic-explaineryoung-disabled-new-zealanders-onthe-barriers-to-a-better-life-122226. [Fecha de consulta: 19 de marzo de 2020]

Carroll, Penelope et al. (2018). Enabling participation for disabled young people: study protocol. BMC Public Health, 18 (1): 712. https://doi.org/10.1186/ s12889-018-5652-x

Chavez, Deborah (1985). Perpetuation of Gender Inequality: A Content Analysis of Comic Strips. Sex Roles, 13 (1/2): 93-102. https://doi.org/10.1007/ BF00287463

Crowther, Gillian (1990). Fieldwork Cartoons. The Cambridge Journal of Anthropology, 14 (2): 57-68.

Denney, Reuel (1957). The astonished muse. Chicago: University of Chicago Press.

Dorfman, Ariel y Mattelart, Armand (1971). Para leer al Pato Donald: comunicación de masa y colonialismo. Valparaíso: Ediciones Universitarias de Valparaíso.

Dorfman, Ariel y Jofré, Manuel (1974). Superman y sus amigos del alma. Buenos Aires: Galerna. 
Eco, Umberto (1968). Apocalípticos e integrados. Barcelona: Lumen.

Ekachai, Daradirek y Drout, Cheryl (1996). Gender Roles in Japanese Comics. Journal of the Illinois Speech and Theatre Association, 47: 19-24.

Frazer, Elizabeth (1987). Teenage girIs reading Jackie. Media Culture and Society, 9 (4): 407-425. https://doi. org/10.1177/016344387009004003

Gabilliet, Jean P. (2009). Of Comics and Men: A Cultural History of American Comic Books. Jackson: University Press of Mississippi. https://doi.org/10.14325/ mississippi/9781604732672.001.0001

Gasca, Luis (1966). Tebeos y cultura de masas. Madrid: Editorial Prensa Española.

Gasca, Luis (1967). Influencia del cómic en la publicidad, Revista Española de la Opinión Pública, 8: 125-142. https:// doi.org/10.2307/40180642

Giroux, Henry (2001). El ratoncito feroz. Disney o el fin de la inocencia. Madrid: Fundación Germán Sánchez Ruipérez.

Glascock, Jack y Preston Schreck, Catherine (2004). Gender and Racial Stereotypes in Daily Newspaper Comics: A Time Honored Tradition? Sex Roles, 51 (7-8): 423-431. https://doi.org/10.1023/B:SERS.0000049231.67432.a9

Groensteen, Thierry (2008). Why are comics still in search of cultural legitimization? En: Jeet Heer y Kent Worcester (eds.) A Comics Studies Reader. Jackson: University Press of Mississippi, pp. 3-11.

Hajdu, David (2009). The ten-cent plague: The great comic-book scare and how it changed America. New York: Farrar, Straus \& Giroux.

Harper, Douglas (2012). Visual Sociology. London: Routledge. https://doi. org/10.4324/9780203872673

Heisler, Florence (1946). A comparison of comic book and non-comic book readers of the elementary school. Journal of Educational Research, 40 (6): 458464. https://doi.org/10.1080/0022067 1.1947.10881536

Hill, George y Trent, M. Estelle (1940). Children's interest in comic strips. Journal of Educational Research, 34 (1): 30-36. https://doi.org/10.1080/00220973.19 41.11010239
Hogben, Lancelot (1949). From cave painting to comic strip; a kaleidoscope of human communication. New York: Chanticleer Press.

Hoult, Thomas (1949). Comic books and juvenile delinquency. Sociology and Social Research, 33: 279-284.

Kendall, Patricia y Wolf, Katherine (1949). The analysis of deviant cases in communications research. En: Paul Lazarsfeld y Frank Stanton (eds.). Communications Research 1948-1949. Nueva York: Harper \& Brothers, pp. 152-179.

Kuschnir, Karina (2016). Ethnographic drawing: eleven benefits of using a sketchbook for fieldwork. Visual Ethnography, 5 (1): 103-134. doi: 10.12835/ ve2016.1-0060

Lazarsfeld, Paul (1940). Radio and the Printed Page: An Introduction to the Study of Radio and Its Role in the Communication of Ideas. Nueva York: DueII, Sloan and Pearce.

Lasswell, Harold (1927). Propaganda Techniques in the World War. London: K. Paul, Trench, Trubner \& Co.

Lent, John (2010). The winding, pot holed road of comic art scholarship. Studies in Comics, 1 (1): 7-33. https://doi. org/10.1386/stic.1.1.7/1

Martín, Antonio (2011). La historieta española de 1900 a 1951. Arbor, 187 (Extra_2): 63-128. https://doi. org/10.3989/arbor.2011.2extran2114

Matacin, Mala y Burger, Jerry (1987). A Content Analysis of Sexual Themes in Playboy Cartoons. Sex Roles, 17 (34): 179-186. https://doi.org/10.1007/ BF00287624

McLuhan, Marshall (1951). The mechanical bride. Folklore of industrial man. New York: Vanguard Press.

Merton, Robert K. (1946). Mass Persuasion: The Social Psychology of a War Bond Drive. Nueva York: Harper \& Brothers.

Miller, Ann (2007). Reading Bande Dessinée: Critical Approaches to French Language Comic Strip. Bristol: Intellect.

Moix, Ramon Terenci (1968). Los "comics". Arte para el consumo y formas pop. Barcelona: Llibres de Sinera.

Murphy, Katherine (2016). Analyzing Female Gender Roles in Marvel Comics from the Silver Age (1960) to the Present. Discussions, 12 (2). Disponible en: http://www.inquiriesjournal.com/ a?id=1449 [Fecha de consulta: 19 de marzo de 2020].

Parasecoli, Fabio (2014). Gluttonous crimes: Chew, comic books, and the ingestion of masculinity. Women's Studies International Forum, 44: 236-246. https://doi. org/10.1016/j.wsif.2013.04.011

Pavlotski, Alex (2015). Visualising Parkour. [Tesis doctoral inédita]. Melbourne: La Trobe University.

Pustz, Matthew (1999). Comic book culture: Fanboys and true believers. Jackson: University Press of Mississippi.

Riesman, David; Glazer, Nathan y Denney, Reuel (1950). The lonely crowd. A Study of the Changing American Character. New Haven: Yale University Press.

Sabin, Roger (1996). Comics, comix \& graphic novels. Londres: Phaidon.

Schultz, Henry (1949). Censorship or self-regulation. The Journal of Educational Sociology, 23 (4): 215-224. https://doi.org/10.2307/2264557

Shaheen, Jack (1994). Arab images in American comic books. Journal of Popular Culture, 28 (1): 123-133. https://doi.org/10.1111/j.00223840.1994.2801_123.x

Simonson, Peter (2006). Introduction. The Annals of the American Academy of Political and Social Science, 608 (1): 6-24. https://doi. org/10.1177/0002716206292527

Smith, Matthew J. (2001). The Tyranny of the Melting Pot Metaphor. En: Matthew McAllister, Edward Sewell y lan Gordon (eds.). Comics \& Ideology. New York: Peter Lang, pp. 129-150.

Smoodin, Eric (ed.) (1994). Disney Discourse: Producing the Magic Kingdom. London: Routledge.

Sousanis, Nick (2015). Unflattening. Cambridge: Harvard University Press.

Steimberg, Oscar (1982). Historieta e ideología en la Argentina: 1936-1937 en la vida de Patoruzú. En Masotta, Oscar (ed.). La historieta en el mundo moderno. Barcelona: Paidós, pp. 159-173.

Stephens Griffin, Nathan (2019). Comics and visual biography: sequen- 
tial art in social research. Visual Studies, 34 (4): 319-335. https://doi. org/10.1080/1472586X.2019.1691940

Strang, Ruth (1943). Why children read the comics. The Elementary School Journal, 43 (6): 336-342. https://doi. org/10.1086/458179

The Project on Disney (1995). Inside the Mouse: Work and Play at Disney World. Durham: Duke University Press. https://doi.org/10.2307/j.ctv11hpnq5

Thompson, John (1990). Ideology and modern culture: critical social theory in the era of mass communication. Stanford: Stanford University Press.

Thorndike, Robert (1941). Words and the comics. The Journal of Experimental Education, 10 (2): 110-113. https://doi. org/10.1080/00220973.1941.11010241

Thrasher, Frederic (1949). The comics and delinquency: cause or scapegoat. The Journal of Educational Sociology, 23 (4): 195-205. https://doi. org/10.2307/2264555
Tilley, Carol (2017). Educating with comics. En Smith, Matthew y Duncan, Randy (eds.). The Secret Origins of Comic Studies. Nueva York: Routledge, pp. 3-11.

Vidal, Jaume (1999). El cómic como vehículo ideológico. En: Nicolás Cortés (coord.). De Yellow Kid a Superman. Una visión social del cómic. Barcelona: Fundació Josep Comaposada, pp. 14-35.

Vidal, Jaume (2002). Alguien tiene que hacerlo, ¿no lo veis? Alguien tiene que salvar el mundo. En Nicolás Cortés y Jaume Vidal (eds). Superhéroes y villanos. Una visión social del cómic. Barcelona: Fundació Josep Comaposada, pp. 11-43.

Vigurs, Katy; Jones, Steven y Harris, Diane (2016). Higher Fees, Higher Debts: Greater Expectations of Graduate Futures? A Research-Informed Comic. Londres: Society for Research into Higher Education.

Wertham, Fredric (1954). Seduction of the Innocent. New York: Rinehart.

Witty, Paul (1941a). Children's interest in reading the comics. The Journal of Ex- perimental Education, 10 (2): 100-104. https://doi.org/10.1080/00220973.19 41.11010239

Witty, Paul (1941b). Reading the comics: A comparative study. The Journal of Experimental Education, 10 (2): 105-109. https://doi.org/10.1080/00220973.19 41.11010240

Wolf, Katherine y Fiske, Marjorie (1949). The children talk about comics. En: Paul Lazarsfeld y Frank Stanton (eds.). Communications Research 1948-1949. New York: Harper \& Brothers, pp. 3-50.

Woo, Benjamin (2011). The Android's Dungeon: comic bookstores, cultural spaces, and the social practices of audiences. Journal of Graphic Novels and Comics, 2 (2): 125-136. https:// doi.org/10.1080/21504857.2011.60 2699

Woo, Benjamin (2012). Understanding understandings of comics: reading and collecting asmedia oriented practices. Participations: Journal of Audience \& Reception Studies, 9 (2): 180-199. 